\title{
Valentina Rapozzi
}

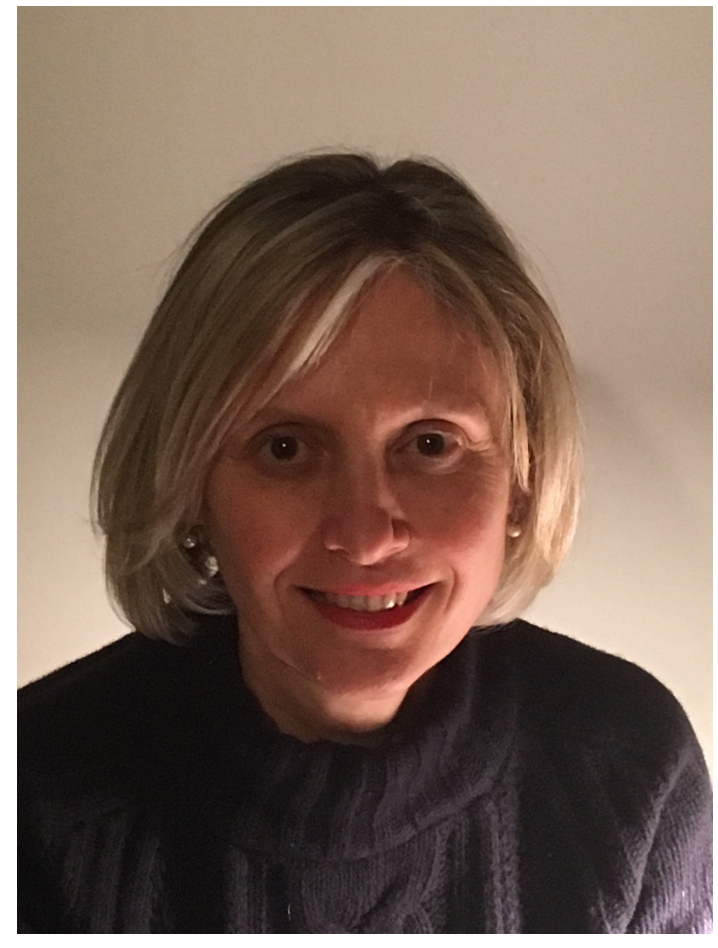

Associate Professor in Biochemistry at the Department of Medicine of the University of Udine. President of the Italian Society of Photobiology.

The research interests of Dr. Rapozzi can be divided into three fields: (1) the application of new photosensitizers in tumor cell cultures in vitro and in vivo systems, and the study of molecular pathways involved in the host's response to photodynamic therapy (PTD); (2) molecular strategies (antigene and antisense) to study neoplastic proliferation; and (3) the influence of psychological stress on metastasis progression in animal models.

Her current research is focused on the study of molecular mechanisms in the tumor response, particularly in the field of redox signaling and photooxidative stress. She is unravelling some mechanisms of the cell response to PDT and their relevance in molecular oncology and cancer. In particular, since 2006, she has contributed to the understanding of the molecular mechanisms involving reactive oxygen and nitric species (ROS/RNS) in coordinating cellular responses to photooxidative stress in different cell/animal models. Her background includes molecular and cellular biology as well as biochemistry techniques. She has participated in several National Research Projects funded by AIRC, PRIN at the University of Udine, and she collaborated with different National Research Institutes such as CNR and other Italian universities.

Dr. Valentina Rapozzi has authored more than 70 publications in international peer reviewed journals as well as 10 book chapters. She was Guest Editor of Forum on Immunopathological Diseases and Therapeutics in 2011 for the volume "Molecular Pathways in the Response of Tumors to Photodynamic Therapy" and Co-Editor alongside Professor Giulio Jori of the University of Padova, Italy, for the volume "Resistance to Photodynamic Therapy in Cancer" published by Springer in 2015. 